MATEC Web of Conferences 8, 01004 (2013)

DOI: $10.1051 /$ matecconf $/ 20130801004$

C Owned by the authors, published by EDP Sciences, 2013

\title{
Simultaneous spatio-temporal focusing for tissue manipulation
}

\author{
J. Squier, E. Block, M. Greco, A. Allende Motz, C. Durfee \\ Center for Microintegrated Optics for Advanced Bioimaging and Control \\ Department of Physics \\ Colorado School of Mines \\ Golden, CO, USA 80401 \\ O. Masihzadeh, D. Ammar, M. Kahook, N. Mandava \\ Department of Ophthalmology \\ University of Colorado School of Medicine \\ Aurora, CO, USA 80045 \\ jsquier@mines.edu
}

\begin{abstract}
Simultaneous spatiotemporal focusing (SSTF) is applied to lens tissue and compared directly with standard femtosecond micromachining of the tissue at the same numerical aperture. Third harmonic generation imaging is used for spatio-temporal characterization of the processing conditions obtained with both a standard and SSTF focus.
\end{abstract}

\section{Simultaneous spatiotemporal focusing with application to micromachining}

Simultaneous spatiotemporal focusing [1-2] enables the precise delivery of energetic pulses as a result of the effective suppression of nonlinear processes such as self-focusing [3-4]. Figure 1 illustrates the dramatic difference between a standard focus (left) and a SSTF focus (right) as function of pulse energy.

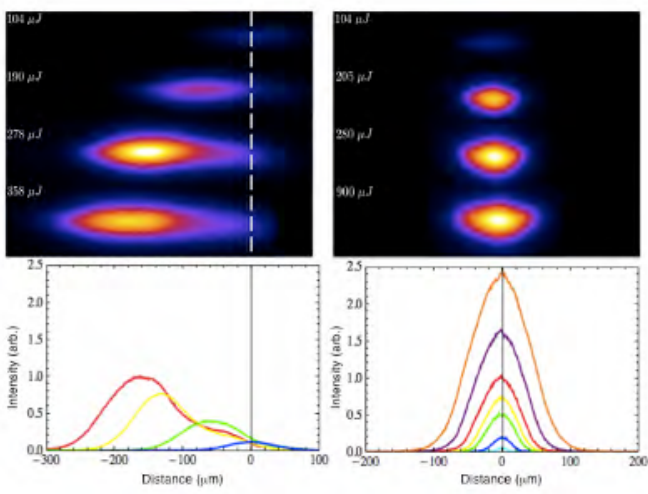

Fig. 1.Comparison of a standard focus (left) and an SSTF focus (right)

In figure 1, the plasma breakdown created at focus is imaged as a function of increasing pulse energy. In the standard case (left column), as the pulse energy is increased the focal spot shifts away from the paraxial image plane and moves toward the focusing optic. Once the energy is increased by $\sim 3.5$ times, the focal spot has shifted by $\sim 200$ $\mu \mathrm{m}$ and is visibly distorted. In contrast, the SSTF focus remains at the paraxial focal plane and is undistorted even when the pulse energy is increased by $\sim 9$ times. This localization makes SSTF much more amenable to large scale tissue manipulation where energetic pulse delivery is desirable as a result of the broad range of tissue conditions and the necessity to maintain precise delivery as a result of having to ablate close to sensitive membranes.

\section{Lens tissue}

This localized behavior is immediately realized as applied to tissue ablation. In figure 2, histological slices of porcine lens tissue ablated with a standard focus and an SSTF focus are compared. In each instance, a $35 \mu \mathrm{m}$ focal spot was directed at the surface of a porcine lens. The pulse duration was $\sim 75 \mathrm{fs}$, the pulse repetition rate is $1 \mathrm{KHz}$, the average laser power is $90 \mathrm{~mW}$, and the sample translation speed was kept constant in all cases. Identical ablation conditions results in significantly different damage morphology. The damage with the standard focus extends for 100's of $\mu \mathrm{m}$ into the sample, while the SSTF ablation zone is limited to the targeted surface of the lens. 


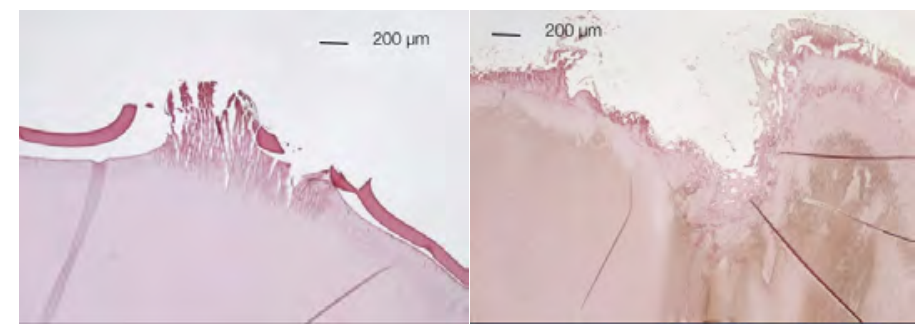

Fig. 2. Comparison of surface ablation of porcine lens using SSTF (left) and a standard focus (right)

\section{Third harmonic generation characterization}

An extremely useful nonlinearity, third harmonic generation (THG), can be used to characterize both the spatial and the temporal characteristics of the SSTF focus. In addition, THG can be used to quantify the sample morphology post processing. THG images comparing SSTF ablation with a standard focus on the front surface, in the bulk, and the back surface of a controlled material will be presented at the conference.

\section{Acknowledgments}

This work was partially supported by the National Institute of Biomedical Imaging and Bioengineering under the Biophotonics Research Partnership grant EB-003832 and partially supported by The US Air Force Office of Scientific Research under program FA9550-12-1-0482.

\section{References}

[1] D. Oron, E. Tal and Y. Silberberg, "Scanningless depth-resolved microscopy," Opt. Express 13, 1468-1476 (2005).

[2] G. Zhu, J. van Howe, M. Durst, W. Zipfel, C. Xu, "Simultaneous spatial and temporal focusing of femtosecond pulses," Opt. Express 13, 2153-2159 (2005).

[3] D. Vitek, D. Adams, A. Johnson, P. Tsai, S. Backus, C. Durfee, D. Kleinfeld, J. Squier, "Temporally focused femtosecond laser pulses for low numerical aperture micromachining through optically transparent materials," Opt. Express 18, 18087-18094 (2010).

[4] D. Vitek, E. Block, Y. Bellouard, D. Adams, S. Backus, C. Durfee, D. Kleinfeld, J. Squier, "Spatio-temporal focused femtosecond laser pulses for nonreciprocal writing in optically transparent materials," Opt. Express 18, 24673-24678 (2010).

[5] C.G. Durfee, M. Greco, E. Block, D. Vitek, and J.A. Squier, "Intuitive analysis of space-time focusing with double ABCD calculation," Opt Express, 20, 14244 (2012). 